\title{
Jóvenes mapuche en movimiento: la metafísica sedentaria en foco
}

Young mapuche on the move: sedentarist metaphysic under the spotlight

\section{Laura Kropff Causa}

\section{(2) OpenEdition}

12 Journals

Edición electrónica

URL: http://journals.openedition.org/aa/3973

DOI: $10.4000 /$ aa.3973

ISSN: 2357-738X

Editor

Programa de Pós-Graduação em Antropologia Social (UnB)

\section{Edición impresa}

Fecha de publicación: 1 diciembre 2019

Paginación: 185-204

ISSN: 0102-4302

\section{Referencia electrónica}

Laura Kropff Causa, «Jóvenes mapuche en movimiento: la metafísica sedentaria en foco», Anuário Antropológico [En línea], v.44 n.2 | 2019, Publicado el 03 diciembre 2019, consultado el 28 abril 2021. URL: http://journals.openedition.org/aa/3973 ; DOI: https://doi.org/10.4000/aa.3973

\section{(c) $\underset{\mathrm{EY} \text { No } \mathrm{ND}}{(\mathrm{O})}$}

Anuário Antropológico is licensed under a Creative Commons Atribuição-Uso Não-Comercial-Proibição de realização de Obras Derivadas 4.0 International. 


\title{
Jóvenes mapuche en movimiento: la metafísica sedentaria en foco
}

\section{Young mapuche on the move: sedentarist metaphysic under the spotlight}

\author{
Laura Kropff Causa \\ IIDyPCa, Universidad Nacional de Río Negro \\ Consejo Nacional de Investigaciones Científicas y Técnicas/CONICET - Argentina
}

\section{Introducción}

En el año 2017, dos sucesos interpelaron fuertemente a la sociedad argentina y a los habitantes de las provincias del norte de la Patagonia en particular: la desaparición y posterior aparición sin vida de Santiago Maldonado en contexto de represión de la Gendarmería Nacional a Pu Lof en Resistencia Cushamen, en la provincia de Chubut, y el asesinato de Rafael Nahuel en contexto de represión por parte de la Prefectura Naval al Lof Lafken Winkul Mapu, en la provincia de Río Negro. En ambos casos se trata de comunidades constituidas, en su mayoría, por jóvenes que habían vivido gran parte de sus vidas en las ciudades de la zona y habían tomado la decisión de recuperar territorio.

Debido al impacto público de estos hechos a nivel nacional e internacional, los investigadores que venimos trabajando, tanto en el estudio del proceso histórico de incorporación de los pueblos indígenas al Estado nación, como en el análisis del activismo mapuche contemporáneo, fuimos interpelados por distintos actores sociales: medios de comunicación; instituciones educativas; organizaciones sociales; comunidades mapuche; etc. Así, temas y problemas que venían siendo periféricos para la opinión pública se colocaron en el centro de la reflexión. En ese marco, fui convocada al debate por mi experiencia de investigación sobre temas vinculados al activismo mapuche y, especialmente, al activismo de los jóvenes urbanos. Uno de los temas puestos en foco fue el de la relación de los jóvenes mapuche con los lugares urbanos y rurales: su posibilidad y su legitimidad.

En ese sentido, en un artículo anterior di cuenta del modo en que la construcción hegemónica de aboriginalidad espacializa subjetividades marcadas como indígenas en el campo, mientras que la construcción hegemónica de edad espacializa subjetividades marcadas como jóvenes en las ciudades, volviendo la combinación 
de ambas dimensiones impensable, desafiante, sospechosa (Kropff, 2011). En esta oportunidad, me interesa profundizar en la dimensión espacial del problema colocando el foco en la cuestión del movimiento. Por un lado, esta situación permite analizar el desplazamiento entre la ciudad y el campo en el presente y su vínculo con el desplazamiento histórico desde el campo hacia la ciudad. Por otra parte, habilita la indagación en relación a la movilidad misma como práctica social estructurada en la intersección entre edad y aboriginalidad. Entonces, comenzaré por dar cuenta del modo en que se produce el desplazamiento de aquellos definidos como "indígenas" en el norte de la Patagonia, en el marco del proceso histórico de consolidación de la matriz Estado-nación-territorio en Argentina. Luego abordaré críticamente la lógica que configura la movilidad como excepción a la luz de la metafísica sedentaria de los estados nacionales, a fin de recuperar un enfoque que la entienda como práctica constitutiva de los procesos de (re)territorialización estatal disputada. Desde esa perspectiva, analizaré la movilidad como una práctica cotidiana estructurada por fricciones diferenciales que se producen en la intersección de edad y aboriginalidad. Para ello, presentaré el caso de la cacería como práctica cotidiana que involucra movilidad y fricciones, a la vez que configura y disputa territorialidad en el ámbito comunitario ${ }^{1}$.

\section{Genocidio y re-territorialización}

El Pueblo Mapuche se define como un pueblo preexistente a los estados nacionales de Chile y Argentina. Las organizaciones supracomunitarias delimitan su territorio ancestral en términos geopolíticos, ubicándolo al sur del río Bío-Bío en Chile y en la Patagonia argentina al sur de las provincias de La Pampa y Buenos Aires (Coordinación de Organizaciones Mapuche Taiñ Kiñe Getuam, 1995). La matriz Estado-nación-territorio se fue consolidando en Argentina hacia fines del siglo XIX, cuando se ocuparon militarmente territorios en los cuales todavía había pueblos autónomos que eran definidos como "indígenas". La conquista militar, que concluyó formalmente en 1885, implicó la ruptura de las formas de organización mapuche y fue la base de una política de usurpación y redistribución de tierras y personas. Se inició así un genocidio que estructuró, de allí en más, las relaciones sociales instaladas en el norte de la Patagonia (Delrio et al., 2018). Se trata de un evento que operó des-territorializando, es decir, rompiendo los códigos de control que regulaban los flujos de acción humana, e impulsando una re-territorialización, es decir, una recodificación de los flujos dominada por una nueva lógica (Deleuze; Guattari, 1987). 
Uno de los aspectos de la re-territorialización estructurada por la lógica estatal genocida es la configuración de fijaciones y movilidades basadas en la organización de accesos diferenciales a la tierra, de acuerdo con un modelo de ciudadanía atravesado por el racismo. Se trata de un modelo que, fundamentándose en características fenotípicas y en prácticas culturales, establece qué pobladores son "deseables" y cuáles "indeseables" para el país moderno que se pretende construir. Entonces, aquellos que son considerados aptos para contratar con el Estado - inmigrantes europeos o sirio-libaneses (y sus descendientes) - obtienen fácilmente títulos de propiedad sobre la tierra. En contraste, aquellos que son considerados como sujetos menos calificados - los definidos como indígenas - únicamente obtienen permisos precarios de ocupación, lo que configura una situación de inestabilidad permanente (Pérez, 2016). En efecto, a lo largo del siglo XX, esa población ha sido objeto de sucesivos desplazamientos que han tenido como destino las ciudades de la región.

$\mathrm{Al}$ mismo tiempo, el proceso de re-territorialización, bajo la lógica del Estado nación, conlleva la configuración de un orden espacial y social específico que implica la consolidación de la ciudad y el campo como lugares diferenciados. La dualidad campo/ciudad emerge de una construcción asimétrica de lugar que se instauró en el proceso de consolidación y expansión del capitalismo en el mundo y que devino lógica explicativa de lo social (Williams, 2001 [1973]). En este caso, esa lógica fue impulsada luego de la ocupación del territorio por parte del Estado argentino. Se trata de una construcción que naturaliza y substancializa las relaciones sociales de producción impuestas por el capitalismo, espacializando subjetividades también asimétricas basadas en la oposición fundante de la modernidad: civilizado/ salvaje (Svampa, 1994). Por tanto, en el desplazamiento hacia la ciudad - que en ese mismo proceso estaba siendo producida - los “indígenas” debían, de alguna manera, dejar su indigeneidad (asociada hegemónicamente al salvajismo) detrás. Esta operación se condice, a su vez, con una lógica estatal asimilacionista.

De hecho, el Estado argentino comenzó a reconocer la presencia de Pueblos Indígenas en su territorio (y su derecho a la identidad y a la tierra), recién en la década de 1990. Hasta la reforma constitucional de 1994, que reconoce la preexistencia étnica y cultural de los Pueblos Indígenas (art. 75, inc. 17), la única mención a los mismos en la carta magna recomendaba mantener un trato pacífico con ellos y promover su conversión al catolicismo. Una vez reconocidos, el Estado se vio en la obligación de saber cuántos eran y dónde estaban ubicados los indígenas. Por ello, en el cuestionario del Censo Nacional de Población y Vivienda del 2001 se incluyó 
por primera vez una pregunta referente a la identidad indígena de la población ${ }^{2}$. Entre los años 2004 y 2005 se realizó la Encuesta Complementaria de Pueblos Indígenas, cuyos resultados incluyen 31 Pueblos. Esa encuesta se basó en los datos del censo nacional 2001 e identificó 113.680 personas que se reconocían descendientes o pertenecientes al Pueblo Mapuche. El 71,6\% de las personas que se reconocían como indígenas en el país se incluía dentro de lo que se consideraba como población urbana. La población total de la provincia de Río Negro, según el censo nacional de 2010, es de 638.645 habitantes y el 9,34\% de los hogares registrados ese año incluyen al menos un integrante que se reconoce descendiente o perteneciente a algún Pueblo Indígena. Sin embargo, considerando la dificultad de implementación del criterio de autorreconocimiento indígena luego de más de un siglo de genocidio y asimilacionismo, sólo podemos tomar estos datos como una referencia que no permite elaborar conclusiones demográficas certeras.

Lo que sí puede afirmarse es que las políticas estatales y las acciones de privados han promovido (y aún promueven) la expulsión de los mapuche de las áreas rurales para favorecer la concentración de tierras en manos de capitales extranjeros y nacionales (para el caso de la provincia de Río Negro, ver Kropff et al., 2019; Ruffini, 2001). Como efecto de este proceso, la población mapuche hoy se concentra mayoritariamente en los barrios marginales de las ciudades que se emplazan en lo que fue territorio autónomo hasta fines del siglo XIX. En cuanto a las áreas denominadas rurales, encontramos que las políticas de radicación redundaron en la configuración de algunos colectivos indígenas que lograron negociar tierras tempranamente (Briones; Delrio, 2002; Delrio, 2005), así como otros colectivos que nunca fueron reconocidos como tales por el Estado. En algunos casos fueron reconocidos como indígenas, pero no como comunidad (Cañuqueo et al., 2015). Aún en los casos en los que hubo concesiones de tierras, la situación a lo largo del siglo XX fue de precariedad en la tenencia debido, por un lado, a la ambigüedad jurídica en relación al derecho de ocupación y, por otro, a prácticas estatales que han contradicho el marco normativo a lo largo de la historia (Martinelli, 2019) ${ }^{3}$.

\section{La movilidad como excepción}

Ahora bien, en relación a la lógica (re)territorializadora de los estados nacionales, distintos autores recuperan el argumento de la antropóloga Liisa Malkki (1992) que sostiene que la misma opera a través de una metafísica sedentaria que hunde personas y culturas en el suelo nacional para segmentar el mundo en unidades 
mutuamente excluyentes representadas en mapas. Para legitimar esta configuración (arbitraria), se apela a metáforas botánicas y parentales: la madre tierra brinda los nutrientes a la patria y la cobija (Alonso, 1994). Desde esta perspectiva, el desplazamiento es concebido en términos de desarraigo botánico, por lo que atenta contra la nación y la cultura a la vez que señala una pérdida moral sumada a la fragilidad emocional (Malkki, 1992). Entonces, a la normalidad de la fijación en un lugar, se opone la anormalidad del desplazamiento.

Por su parte, quienes abordan movilidades en relación a Pueblos Indígenas en el mundo, se encuentran con los efectos del nativismo como discurso informado por la metafísica sedentaria. El nativismo otorga autenticidad a aquellos pueblos enraizados (botánicamente) en el suelo de la nación, antes de que la propia nación exista. En esa clave, cualquier experiencia de movimiento supone necesariamente la disolución de la identidad, la aculturación y la asimilación (Clifford, 2007). Así, la lógica de (re)territorialización estatal argentina aplicada en la Patagonia configura una paradoja: a la vez que obliga a los indígenas a moverse reiteradamente, considera la movilidad una práctica excepcional opuesta a los criterios que definen tanto la identidad nacional como la autenticidad étnica.

Ahora bien, las movilidades y fijaciones diferenciales asociadas a la configuración de ciudadanía no operan únicamente en clave de aboriginalidad (Beckett, 1988; Briones, 1998), sino que se despliegan articulando marginaciones múltiples en la intersección de los distintos clivajes que estructuran la práctica social: etnicidad; clase; género; nacionalidad; etc. (ver, entre otros, Dhamoon, 2011; Hancock 2007). Uno de esos otros clivajes - líneas que estructuran la organización social estableciendo dinámicas de agregación y desagregación (Briones; Siffredi, 1989) - es la $\operatorname{edad}^{4}$. Al tiempo que las subjetividades marcadas como indígenas se espacializan en el campo, las subjetividades marcadas como jóvenes se espacializan en las ciudades y esta topología deviene una cronotopología (Durham, 2004), al asignar a cada una de estas subjetividades espacializadas una porción de tiempo. Mientras a los indígenas ruralizados se les asigna el pasado, a los jóvenes urbanizados se les asigna el futuro. El presente sigue siendo prerrogativa de una subjetividad desmarcada tanto en términos étnicos como en términos etarios y, por lo tanto, ubicada por fuera del esquema espacializador. En tanto, como sugiere Slavoj Zizek (1998), los procesos de des-marcación conllevan la universalización de una particularidad que se constituye en enunciadora y que marca las particularidades, las "minorías", las "parcialidades”. En este caso, esa particularidad dueña del presente es adulta y no es indígena. 
Esta construcción configura al joven mapuche como una subjetividad escindida tanto en el espacio como en el tiempo y, por ello, impensable, desafiante y/o sospechosa. Como se vio en el caso de Rafael Nahuel que mencioné en la introducción, se trata también de una subjetividad matable (Agamben, 1998).

Asociadas a estas subjetividades espacializadas se encuentran sus posibilidades de movimiento. Así, decía en 2011 que en el contexto etnográfico de los parajes rurales de la Línea Sur rionegrina ${ }^{5}$, la única posibilidad de visibilización de los jóvenes se constituye en el acto de irse. "Los jóvenes se van” es una frase reiterada tanto por los pobladores como por los referentes de las instituciones estatales que se desempeñan en la zona. De este modo, el retorno de los jóvenes se constituye en utopía y de ella se hacen cargo los jóvenes mapuche nacidos en las ciudades que buscan articularse con las comunidades configuradas en zonas rurales (Kropff, 2011). Se trata de un tránsito cargado de demandas, responsabilidades y valores morales que no muchos consiguen atravesar. Esta doble vía de la partida y el retorno se constituye como única posibilidad de tránsito (y de movimiento en general). Así, el telón de fondo de la espacialización hegemónica de aboriginalidad y edad es la metafísica sedentaria que se traduce en un intenso trabajo tendiente a configurar la fijación como parámetro y la movilidad como excepción. Esa construcción se constituye como interpelación sobre prácticas de ocupación y producción de sentido en torno al espacio que no se desarrollan en esos términos, incluyendo las presiones políticas y económicas para el desplazamiento selectivo de las que el propio Estado participa.

\section{Fricciones diferenciales}

Para Mimi Sheller y John Urry, en contraste con la metafísica sedentaria, la perspectiva fundada en la metafísica nómada elogia la movilidad contemporánea a nivel global como un proceso de desterritorialización que pregona el fin del Estado como contenedor de las sociedades y la liberación de la gente del constreñimiento provocado por el espacio y el lugar (ver, entre otros, Bauman, 2003). En ese sentido, las metáforas del viaje y el vuelo celebran lo que se entiende como disolución de las fronteras estatales. Sin embargo, esta perspectiva constituye una antítesis que da por sentado el hecho de que las fronteras y la fijación son una realidad objetiva y no el producto de una operación sociocultural y política históricamente sedimentada. A partir del cuestionamiento tanto a la metafísica sedentaria como a la metafísica nómada, el enfoque centrado en la movilidad la entiende como práctica localizada 
que, en todo caso, reorganiza la materialidad de los lugares (Sheller; Urry, 2006). Esta perspectiva abre la pregunta por el modo diferencial en que se configuran tanto la movilidad como la estabilidad a través de discursos y prácticas, sin dar por sentado el vínculo entre gente, lugar y cultura que se propone tanto en clave sedentaria como en clave nómada. Más que celebrar el movimiento sin límites, el foco está en los procesos económicos y políticos por los que se establecen los límites y la gente es ubicada y/o se le permite o fuerza al movimiento.

En este marco, Noel Salazar y Alan Smart recuperan el concepto de "fricción" de Anna Lowenhaupt Tsing (2005), que aplica a los procesos sociales la noción física de que la aceleración siempre confronta con una fuerza que tiende a frenarla, a la vez que habilita el movimiento. Los autores piensan a la movilidad como productora de alteridad en la generación de desigualdades de velocidad, riesgo, derecho y estatus. En ese sentido, no hay una clara separación entre elección y constreñimiento, movilidad voluntaria o forzada. La movilidad crea tensiones y las trayectorias de movimiento que adoptan los individuos y los grupos dan forma tanto a su vida como a los lugares que habitan (Salazar; Smart, 2011). Ya en 1963, Roberto Cardoso de Oliveira apelaba al término "fricción” para dar cuenta de la dinámica de las relaciones interétnicas en situaciones de contacto que suponen asimetría y violencia, particularmente en el marco de los procesos de expansión de los estados nacionales sobre territorios indígenas (Cardoso de Oliveira, 1963). Recuperando ambas perspectivas, se puede pensar al genocidio indígena como un evento con consecuencias en la definición racializada y etnicizada de movilidades y fijaciones diferenciales en la lógica de re-territorialización estatal ${ }^{6}$.

Según James Clifford, la oposición hegemónicamente determinada entre la experiencia indígena y la movilidad no permite entender cómo estos pueblos han producido sentido en relación a las experiencias de genocidio, desposesión material, asimilación forzada, marginalidad (política, cultural, racial y económica). Esa producción de sentido conlleva oportunidades para el cambio y la re-identificación por lo que el autor sostiene que se puede hablar de dimensiones diaspóricas de la experiencia indígena, entendidas como aspectos de un continuum de apegos desiguales (Clifford, 2007). De hecho, los intelectuales orgánicos del movimiento mapuche han comenzado a acuñar el término "diáspora" para referirse a la des-vinculación con el territorio. Cuando lo que se analiza es la consolidación de la matriz Estado-nación-territorio en Chile, es la migración a la ciudad de Santiago la que se califica con diáspora, entendiendo el territorio de origen en términos geopolíticos 
e históricos, como aquel que se encuentra al sur del río Bío (Ancan; Calfío, 2002). El concepto de diáspora se define aquí como un exilio partiendo de una noción de territorio que evoca la metafísica sedentaria de los estados nacionales, esto es: un territorio con fronteras bien delimitadas que se entiende homogéneo al interior. La reflexión política producida en torno a estos desplazamientos para el caso chileno ha llevado a referentes mapuche de Argentina a pensar la diáspora como metáfora para explicar la trashumancia dentro del territorio ancestral, denominadoWajmapu, pero apropiado por otros. Es decir que la distancia, desde esta perspectiva (compartida en conversaciones informales), está producida por la imposibilidad de ocupar los espacios y determinar con autonomía lo que en ellos ocurre.

Por su parte, Ana Vivaldi plantea que la movilidad no puede pensarse como un único viaje migratorio, ya que implica idas y vueltas constantes. Así, los movimientos que dan forma a los lugares no son sólo los flujos transnacionales o los desplazamientos unidireccionales entre lo rural y lo urbano, sino también prácticas de movilidad a pequeña escala que conllevan fricciones particulares en la cotidianeidad. De este modo, abre la pregunta por los tipos interconectados de movimientos y fricciones como clave para dar cuenta de los procesos que están operando en la espacialización de subjetividades indígenas en el presente (Vivaldi, 2011). Esta perspectiva permite, a su vez, la indagación sobre los movimientos y fricciones que se dan en la intersección entre aboriginalidad y edad en la vida cotidiana.

Entre otros ejemplos de movilidad cotidiana, durante mi trabajo de campo en parajes rurales de la provincia de Río Negro pude identificar circuitos que se perfilan en los márgenes de las movilidades estructuradas por las categorías “joven” y "mapuche". Se trata de experiencias de muchachos que pasan algunas temporadas en el campo ocupándose de los animales (pero yendo y viniendo del pueblo), otras en la ciudad y otras transitando diferentes parajes y provincias en comparsas de esquila. En algunos contextos se definen y son definidos como jóvenes y en otros no. Quizás se reconocen como "paisanos", pero "mapuche" es una categoría cargada de sentidos politizados que les resultan ajenos, aunque sus abuelas hablen en mapuzugun (idioma mapuche). Es un circuito eminentemente masculinizado, y es en clave de género que se vuelve visible. Sin embargo, es elusivo para categorías fijadoras que, tanto en clave de edad como de etnicidad, consideran los desplazamientos como momentos excepcionales. 


\section{Movilidad y fricción en las prácticas comunitarias: la cacería como ejemplo}

Desde que comencé a hacer trabajo de campo con jóvenes mapuche urbanos en 2001, fui testigo de distintas escenas de intercambio de regalos vinculados a la caza de animales silvestres. Eventualmente, yo misma recibí plumas de choike (ave similar al avestruz) cuando entablé amistad con una familia en particular. Cuando, en 2008, comencé a recorrer parajes rurales de la cuenca del arroyo Comallo en la Línea sur de la provincia de Río Negro, empecé a escuchar relatos y bromas vinculados a la cacería. Generalmente se trataba de comentarios que los hombres se hacían entre sí y que no involucraban a las mujeres. De alguna manera la caza estaba relacionada con el establecimiento de vínculos asegurados por el intercambio de regalos - en el sentido de la noción clásica de intercambio de dones (Mauss, 1979 [19234]) - pero también con otras cosas. Desde la perspectiva de quienes participan de la actividad, también en las juntadas para "bolear" (término nativo para referirse a la cacería) se fortalecen los vínculos. Un integrante de la comunidad mapuche Newen Ñuke Mapu (Fuerza de la Madre Tierra), me decía al respecto:

En las boleadas nosotros nos juntábamos y terminábamos en una casa jugando al futbol. (...) Entonces algunos sabían estar preparando unas zapatillas viejas para jugar al futbol. Salían a la boleada, sí, pero lo que querían era jugar al futbol. (...) La cosa de pasar juntos jodiendo un buen rato y hacerse chistes porque se le fue el zorro, porque no... alguno que se enojaba porque el otro no estaba o porque algunos se juntaban para charlar en vez de estar mirando los zorros.

Así, la caza colectiva se convierte en un importante espacio de socialización entre los hombres viejos y jóvenes de los parajes rurales que, desde 2011, integran formalmente la comunidad Newen Ñuke $\mathrm{Mapu}^{7}$. Asimismo, se trata de una práctica que implica la configuración de territorio a través del movimiento. La corrida de zorros, especialmente, implica atravesar distintos campos y esto se realiza pidiendo permiso a los pobladores, una fricción habilitadora del movimiento. En general los permisos se otorgan sin problemas excepto en el caso de "los foráneos". El límite del territorio de caza es el límite de los permisos. El conflicto se presenta cuando "los foráneos" se instalan dentro del territorio en el que, históricamente, los permisos estaban garantizados. Aquí es donde se puede observar una nueva fricción en la que resuena la noción de fricción interétnica de Roberto Cardoso de Oliveira (1963). 
Lo que fue limitando la cacería fueron los alambres y la gente que empezó a prohibir la caza. Generalmente prohíben los foráneos: gringos. A veces no gringos pero que vienen de otro lado a invertir específicamente en ovejas o en vacas. Tienen un campo para producir con vacas o con ovejas. (...) El que viene de afuera ya tiene esa desconfianza y, como viene de afuera, tiene otra manera de plantarse (...) Entonces esa gente que tiene más plata tiene temor a que le roben entonces prohíbe la entrada y prohíbe cazar. Vos podés andar por ahí y vas a ver un cartel que dice "prohibido cazar". Uno incluso puso un cartel que decía "Área privada protegida” (Cazador de la NÑM).

De este modo, la distinción entre propios y ajenos se va inscribiendo en el uso del espacio. Lo que antes de la llegada de los privados constituía un lugar de tránsito para la boleada colectiva de zorro, se convierte en un "área privada protegida”. La consecuencia es el entorpecimiento, no solamente de la persecución de un predador que afecta la producción ganadera en una zona donde la economía se basa en la cría de ganado ovino y caprino, sino del desarrollo de las estructuras sociales de relación asociadas a esta práctica comunitaria. Así, este proceso constituye un ejemplo de la fricción que se genera sobre la movilidad cotidiana como ejercicio continúo producido tanto por agencias estatales como por actores "foráneos" que aplican la lógica de la demarcación de la propiedad privada.

En contraste, la práctica de la caza colectiva pone en evidencia una noción de ocupación territorial basada en los recorridos, el tránsito (ver, también, Cañuqueo, 2015). En estos recorridos se comparten conocimientos específicos sobre el paisaje, la vegetación, los animales, los fenómenos meteorológicos, etc. Además, implican la transmisión de valores y destrezas entre hombres de distintas generaciones y, en ese sentido, constituyen experiencias formativas. Se trata de una movilidad configurada a través de relaciones sociales puestas en evidencia en la solicitud de permisos - la fricción de los propios - y sostenidas por el intercambio de presas como regalos que no se circunscriben a lo que se entiende como espacio rural sino que circulan por los espacios de desplazamiento comunitario, incluyendo los barrios periféricos de las ciudades. Esta movilidad de la boleada entró en fricción, en primer lugar, con los alambres que fueron atravesando lo que era "campo abierto". En segundo lugar, la movilidad se vio afectada por la venta de tierras a privados ajenos a los parajes que comenzaron a prohibirla en sus campos.

La relación entre la boleada y el espacio se fue reconfigurando a través de la historia. En un primer momento, el campo abierto demandaba cotidianamente el 
trabajo en colaboración con los vecinos, no sólo para las boleadas, sino para la producción ganadera misma. Era frecuente la necesidad de separar animales, de rastrear algunos que se perdían, etc.

Me acuerdo cuando chico corríamos yeguas acá. Que te traen las yeguas mansas al corral, pero, como son yeguas, siempre tienen el instinto de disparar. $\mathrm{Y}$ al ver movimiento, varias personas, tratan de disparar. Entonces se necesitan varios jinetes para atajarlas y traerlas al corral. Eso se hacía así porque era campo abierto y al ser campo abierto por ahí se mezclaban con las yeguas de los vecinos (Cazador de la NÑM).

El avance de los alambres vino de la mano de la compra, muchas veces fraudulenta, de campos por parte de privados que luego no ponían a producir la tierra, sino que la mantenían en función de la especulación inmobiliaria. Esto acabó teniendo dos efectos: por un lado, los animales de los pobladores continuaron ocupando el espacio que estaban acostumbrados a ocupar y, por otro, proliferaron las yeguas matreras que se incorporaron tanto a la producción ganadera como a la boleada comunitaria. En el presente, grandes extensiones de tierra que la comunidad ocupa tanto con ganado como para bolear son reclamadas por privados y sociedades anónimas que dicen haberla adquirido. La comunidad ha sufrido desalojos y otras presiones. Por supuesto, esto afecta la práctica de la boleada, entre otras actividades comunitarias.

Los chicos [de una de las familias del paraje] cuidan chivas y antes boleaban yeguas. Su actividad económica principal pasaba por eso. Me acuerdo de ir a una marcación y tenían 70 yeguas en el rodeo, en el 99 por ahí. Con la venta que hizo [un terrateniente especulador a un privado] empezaron a alambrar y a decir "acá no porque ahora nosotros compramos acá y esto es propiedad privada” y todas esas cosas. Se empezó a cortar de a poco. No fue algo de un día para otro. O que lo empezaron a ocupar para trabajar y que los fueron presionando. Entonces se fue restringiendo el ingreso.

El territorio definido para la boleada es sólo una parte del territorio de la comunidad Newen Nuke Mapu, ya que no todos los parajes que la integran son cazadores. Sin embargo, los recorridos son vastos, llegando a cubrir hasta alrededor de cuarenta kilómetros, especialmente en las corridas de zorro. 
Dura un día por lo general. Es como en esas películas inglesas. La salida es antes de que cante el gallo. (...) Salíamos de noche para agarrarlo cuando vuelve de su faena. Si comió está en desventaja porque va lleno y cansado porque su horario de dormir es el día. Cuando sale el sol es cuando se va a echar a dormir. La mayoría de las veces no agarrábamos nada, pero veíamos rastro y le pegábamos un julepe... Le marcábamos el territorio. Lo ahuyentábamos. (...) Llevábamos carne, algunos llevaban torta o pan. Y al mediodía se para a hacer un asado en cualquier lado y... descanso un rato y después a continuar. Siempre hacíamos eso porque cubríamos mucha distancia porque el zorro es caminador. Entonces para eso tenemos que traspasar campos de uno, de otro (Cazador de la NÑM).

Lejos de tratarse de un simple recorrido libre por los campos, el desplazamiento implica un recorrido por la trama social de la comunidad. Con lo cual, cuando se limita ese tránsito, se incide también sobre esas relaciones sociales que son constitutivas de la vida comunitaria. Al mismo tiempo, opera en la configuración de subjetividades entramando distintos clivajes, entre ellos la edad:

Y cuando [los grandes] salían a correr zorros. Y a mí me entusiasmaba la idea de ir, pero no me llevaban. En las corridas de yeguas igual.Y se juntaban un montón. (...) A mí lo que me entusiasmaba de eso era el hecho de correr y preparar los animales. Lo lindo que tiene la cacería es eso, la cosa previa, prepararse. Porque vas a ir a exigirte. Si querés tener éxito tenés que estar preparado. Entonces, toda esa cosa previa, más preparase con caballos de reserva. Me acuerdo que llegaban acá y andaban entusiasmadísimos con eso, que les puede llevar dos días. Y con los caballos re-sudados, agitados. Y decían "no, se me fue para tal parte y el otro se fue para allá y este boludo que no volvió y aquellos que estaban mirando. Traeme el otro caballo que voy a seguir."Y entonces cambiaban caballo. Y toda esa efervescencia que había y toda esa adrenalina. Y yo quería participar de eso y no me llevaron porque como había mucha exigencia física y mucho roce físico había que cuidar al chico.

Finalmente, cuando los mayores consideraron que estaba en condiciones de participar, lo incorporaron en las boleadas de zorro:

Hacíamos como un cerco, que es toda una estrategia de ir uno, por un lado, el otro por otro. Ir cercándolo. En una oportunidad uno quedó en una barda. Pasaron un montón de personas para un lado y el zorro, como es zorro, no dispara. Ve, olfatea, escucha, y volvió. Y en esa vuelta queda en una barda donde estoy yo y, al verme a mí, queda y yo justo lo veo y andaba con el rifle. Así que ahí le pegué un tiro.Y, bueno, después de esa, con uno nomás que cacé, cada vez que se hacía una boleada el que organizaba [un 
hombre grande] me venía a buscar (...)Y entonces armaba todo el cerco y me decía 'vos vení acá'. Me daba un lugar especial porque por ahí se podía aparecer.

En la lógica del relato, al cumplir exitosamente con la destreza y el compromiso requerido, al cazador joven se le dio un lugar central en la estrategia de la boleada. Así se lo reconoció y se ganó el respeto de pares y mayores. De este modo, la boleada constituye también un espacio de transmisión intergeneracional de conocimientos y valores entre los hombres. Sin embargo, además de tratarse de una actividad que involucra valores morales, la boleada constituye un espacio lúdico:

Quedó la costumbre en esa segunda generación donde yo estaba, que ya éramos más informales si se quiere, para un montón de cosas. Los otros eran más tradicionales y eran más capos. Claro, con sus caballos, sus sogas, sus perros preparados. Nosotros no éramos tan preparados. Incluso se nos iban porque... porque hacíamos cualquier cosa. Nos poníamos a gritar y no había que gritar en ese momento (...) Nosotros no éramos exigentes por eso no nos importaba si cazábamos algo o no. Y en esa por ahí alguno cazaba un choike y se compartía (...) Éramos una camada de muchachones locos. "Todos locos" decía un vecino. Y él también se prendía en esa. Como cachorros. De repente veníamos de vuelta y nos veníamos molestando los caballos. O nos armábamos... de repente uno salía “y quién llega último a preparar el asado" y salían todos corriendo. Esas cosas los otros creo que no la hacían. (...) Y así cazando creo que anduvimos por toda esta zona.

La boleada deviene un espacio en el que se pone en juego la construcción de masculinidad a través de un modelo basado en la destreza, el compromiso con el trabajo, los juegos y las bromas. Además, constituye un diacrítico que define identidades territoriales primarias ancladas en los parajes y distingue a los propios de los “foráneos". Finalmente, no sólo el género y la etnicidad se ponen en juego en la boleada, sino también la edad, ya que la transmisión de conocimientos y la puesta en acto de valores implica la instauración de la jerarquía etaria. Por un lado, se establece que los niños no pueden participar de la práctica. Por otro, los mayores son quienes organizan y asignan lugares a los jóvenes en la tarea. Finalmente, aparece una distinción entre generaciones: las de antes (los "más capos") y la de ahora (los “cachorros”). Entonces, a través de esa práctica se (re)construye la teoría de la edad propia de ese contexto social. La caza colectiva comunitaria se convierte, por lo tanto, en una práctica que articula el clivaje de género, el étnico y el etario a través 
de una movilidad de pequeña escala que entra en fricción con la ejecución del plan de fijación configurado por la metafísica sedentaria del Estado nación.

\section{Conclusiones}

En este artículo me propuse poner el foco en la movilidad que se estructura en la intersección de aboriginalidad y edad (y también género), para poner en evidencia histórica y etnográfica las fricciones que provoca la metafísica sedentaria del Estado nación en el norte de la Patagonia en Argentina. En ese sentido, la construcción de la movilidad como excepción conlleva la sedimentación de la fijación como parámetro revelando el éxito de un trabajo cultural en permanente proceso: el que vincula naciones (homogéneas) con territorios (delimitados). Así, la codificación de los flujos de la acción humana impulsada por la lógica estatal capitalista redunda en cartografías fijadoras en las que se establecen tránsitos específicos para los jóvenes, para los mapuche y para los jóvenes mapuche.

En cuanto a los mapuche, el genocidio indígena estableció la definición racializada y etnicizada de movilidades dificultando la permanencia en la tierra rural y negándoles, a la vez, la posibilidad de existencia en las ciudades. De modo que el único camino posible es la asimilación, la disolución de su identidad específica dentro de la nación a la vez que se consolida una noción geopolítica de territorio nacional articulada con un espacio productivo definido por la dualidad entre lo rural y lo urbano. En ese marco, los jóvenes pueden moverse en el camino establecido entre el campo y la ciudad (de ida) y entre la ciudad y el campo (de vuelta y utópico), un recorrido habilitado sólo de manera excepcional. Por ello, los múltiples tránsitos que se observan por fuera de este esquema sedimentado configuran movilidades que devienen subalternas e inenarrables.

Deborah Durham (2004) definió el concepto de cronotopología para referirse a aquello que se indexicaliza en el uso del término "juventud”. Su idea era vincular la dimensión temporal con la metáfora del paisaje social. Se trata de una idea potente cuando analizamos la edad imbricada con la aboriginalidad porque el cronos nos remite al proceso histórico de consolidación de la matriz Estado-nación-territorio y la topología cobra una dimensión específica a través del territorio que vimos configurado y disputado aquí en la movilidad cotidiana que implica la cacería. Así, la topología móvil que se produce a través de la cacería se ve atravesada por las fricciones provocadas por la acción tanto de agencias estatales como de actores "foráneos" que se instalan con lógicas de ocupación fundadas en la propiedad privada. 
La apuesta argumentativa de este artículo fue invertir la cartografía hegemónica y destacar, sobre el telón de fondo de la movilidad, el trabajo cultural y político que, informado por la metafísica sedentaria, tiende a la fijación de subjetividades demarcadas en términos étnicos y etarios, provocando diferentes fricciones. Es a través de ellas que, en definitiva, edad y aboriginalidad se configuran y disputan permanentemente.

Recebido: 30/04/2019

Aprovado: 28/08/2019

Laura Kropff Causa es Profesora, Licenciada y Doctora en Antropología formada en la Universidad de Buenos Aires. Actualmente es Investigadora en el Consejo Nacional de Investigaciones Científicas y Técnicas (CONICET) y Profesora en la Universidad Nacional de Río Negro (UNRN) con lugar de trabajo en el Instituto de Investigaciones en Diversidad Cultural y Procesos de Cambio (UNRN-CONICET) en Bariloche, Argentina. Desde 1997 hace trabajo de campo en localidades rurales y urbanas de Nor-Patagonia. ORCID: https: / / orcid.org/0000-0003-20163176. Contacto: kropff@unrn.edu.ar

\section{Notas}

1. Este artículo se basa de una investigación que se enmarca en dos proyectos: el PI-UNRN 40-B637 "El desplazamiento a las ciudades como efecto del genocidio indígena: una aproximación etnográfica al caso de Bariloche" financiado por la Universidad Nacional de Río Negro, y el PICT 2017-1706 "Conflictos por el acceso a la tierra en la provincia de Río Negro: un abordaje etnográfico e histórico a la territorialización de formaciones sociales de alteridad” financiado por el Fondo para la Investigación Científica y Tecnológica de la Agencia Nacional de Promoción Científica y Tecnológica.

2. El criterio que debía utilizarse para medir la denominada "variable indígena" era el de autoreconocimiento y no criterios biologicistas o culturalistas. Por lo tanto, teniendo en cuenta que la construcción de identidades es consecuencia de un proceso relacional y que, en este caso, se trataba de una relación históricamente asimétrica, el censo propuso una situación compleja. El Estado argentino reconoció por primera vez tener población indígena, aún después de más de cien años 
de una política negadora y derogatoria. Ante esto, varias organizaciones mapuche advirtieron que el número resultante no daría cuenta de la cantidad de indígenas sino de los efectos de las políticas extincionistas y asimilacionistas del propio Estado nacional (Organización Mapuche Newentuayiñ y $\mathrm{Pu}$ weche fiske menuko, 2001).

3. Para un análisis de los procesos de territorialización desde una perspectiva teórica similar en Brasil, ver Pacheco de Oliveira, 1998.

4. Abordar la edad como dimensión estructurante de la práctica social conlleva prestar atención, en primer lugar, a las teorías sociales de la edad operantes en cada contexto. Estas teorías configuran las cronologías de los cursos de vida, las categorías que los estructuran, los significados de esas categorías y las relaciones que se establecen entre ellas. En segundo lugar, desde una perspectiva diacrónica, conlleva prestar atención a la configuración de generaciones. Generación es un término polisémico que, por un lado, refiere a los desplazamientos en la experiencia que acaban creando cohortes con conciencia de edad (Comaroff y Comaroff, 1999), lo que los antropólogos clásicos llaman "grupos de edad" (Radcliffe-Brown, 1929). En tanto grupos de edad que atraviesan categorías (o grados) de edad, la dinámica de las generaciones produce sentido en torno al flujo de la experiencia social, otorgando interpretaciones que fijan coordenadas temporales para marcar continuidades y rupturas en el(los) sentido(s) de devenir (Kropff, 2016). El otro campo de sentidos que confluye en el término generación es el de la genealogía, más vinculado al lenguaje del parentesco. Esta ambivalencia permite explorar la confluencia entre parentesco, política y dinámicas históricas en los grupos humanos. Desde esta perspectiva, edad es una categoría teórica englobadora, como lo son aboriginalidad, clase, género, raza, etc.

5. La región denominada Línea Sur, definida por el recorrido de la línea ferroviaria que atraviesa la provincia desde el océano Atlántico hasta la cordillera de los Andes, ha sido históricamente un espacio de radicación de familias y comunidades mapuche dedicadas a la cría de ganado lanar.

6. Con el término "racialización", Briones (2002) busca poner en evidencia los procesos de construcción de alteridades que se naturalizan a partir de su asociación con características que se definen como biológicas y, por lo tanto, inmodificables. De este modo, pone en evidencia que las razas no constituyen entidades definidas por la naturaleza sino efectos de estas operaciones de marcación social de la alteridad. En el mismo sentido, "etnicización” refiere a la operación que asocia subjetividades sociales con características culturales que configuran modos de operar en el mundo que son difíciles de modificar.

7. La Newen Nuke Mapu (Fuerza de la Madre Tierra) se constituyó formalmente como comunidad mapuche en agosto de 2011. Nuclea a más de cuarenta familias de seis parajes rurales de la cuenca del arroyo Comallo en Río Negro. Desde el momento de su constitución formal, uno de los temas centrales de la agenda de la comunidad es la cuestión territorial. Si bien varios de los pobladores consiguieron títulos individuales de propiedad en la década de 1980, otros tantos tienen permiso de ocupación precaria de tierras fiscales y/o conflictos con privados que pretenden desalojarlos de las tierras que ocupan. A esto se suma la preocupación por la posibilidad de realización de prospecciones mineras en la zona. 


\section{Referências}

AGAMBEN, Giorgio. 1998. Homo Sacer: el poder soberano y la nuda vida. Madrid: Pretextos.

ALONSO, Ana. 1994. "The Politics of Space, Time and Substance: State Formation, Nationalism, and Ethnicity”. Annual Review of Anthropology, n. 23, p. 379-405.

ANCÁN, José; CALFÍO, Margarita. 2002. "Retorno al país mapuche. Reflexiones sobre una utopía por construir”. Nuke Mapu förlaget. Working Paper Series 6. Disponible en: http: / /www.mapuche. info/wps_pdf/ankalfio020300.pdf. Acceso en: 28 marzo. 2016.

BAUMAN, Zygmunt. 2003. Modernidad líquida. México: Fondo de Cultura Económica.

BECKETT, Jeremy (ed.). 1988. Past and present. The construction of aboriginality. Canberra: Aboriginal Studies Press.

BRIONES, Claudia. 1998. La alteridad del “Cuarto Mundo”: una deconstrucción antropológica de la diferencia. Buenos Aires: Ediciones del Sol.

BRIONES, Claudia. 2002. "Mestizaje y blanqueamiento como coordenadas de aboriginalidad y nación en Argentina”. Runa, n. 23, p. 61-88.

BRIONES, Claudia; DELRIO, Walter. 2002. "Patria sí, Colonias también. Estrategias diferenciales de radicación de indígenas en Pampa y Patagonia (1885-1900)”. En: TERUEL, Ana; LACARRIEU, Mónica; JEREZ, Omar (Eds.). Fronteras, ciudades y estados. Tomo I. Córdoba: Alción. p. 45-78.

BRIONES, Claudia; SIFFREDI, Alejandra. 1989. "Discusión introductoria sobre los límites teóricos de lo étnico". Cuadernos de Antropología de la Universidad de Luján, n. 3, p. 5-24.

CAÑUQUEO, Lorena. 2015. "El territorio relevado, el territorio disputado: apuntes sobre la implementación de Ley Nacional 26.160 en Río Negro, Argentina”. Revista de Geografía Norte Grande, n. 62, p. 11-28.

CAÑUQUEO, Lorena; KROPFF, Laura; PÉREZ, Pilar. 2015. “A la sombra del Estado: comunalización indígena en parajes de la pre cordillera de Río Negro, Argentina”. Revista del Museo de Antropología, v. 8, n. 2, p. 159-170.

CARDOSO DE OLIVEIRA, Roberto. 1963. “Aculturación y 'fricción' interétnica”. América Latina, v. 6, n. 3, p. 33-46.

COMAROFF, Jean; COMAROFF, John L. 1999. "Occult economies and the violence of abstraction: Notes from the South African postcolony”. American Ethnologist, v. 26, n. 2, p. 279-303.

CLIFFORD, James. 2007. "Varieties of indigenous experience: diasporas, homelands, sovereignties”. En: DE LA CADENA, Marisol; STARN, Orin (Eds.). Indigenous experience today. Oxford; New York: Berg Publications. p. 197-224.

COORDINACION DE ORGANIZACIONES MAPUCHE TAIN KIÑE GETUAM - PARA VOLVER A SER UNO. 1995. “Autonomía y pueblos originarios”. Primer Seminario Regional El Derecho Internacional y los Pueblos Originarios. Neuquén, 29 de septiembre al 2 de octubre, Universidad Nacional del Comahue. Mimeo.

DELEUZE, Gilles; GUATTARI, Félix. 1987 [1980]. A thousand plateaus: capitalism and schizophrenia. Minneapolis: University of Minnesota Press.

DELRIO, Walter. 2005. Memorias de expropiación: sometimiento e incorporación indígena en la Patagonia - 1872-1943. Bernal: Universidad Nacional de Quilmes. 
DELRIO, Walter; ESCOLAR, Diego; LENTON, Diana; MALVESTITTI, Marisa (Eds.). 2018. En el país de nomeacuerdo: archivos y memorias del genocidio del Estado argentino sobre los pueblos originarios, 1870-1950. Viedma: Editorial UNRN.

DHAMOON, Rita Kaur. 2011. “Considerations on mainstreaming intersectionality”. Political Research Quarterly, v. 64, n. 1, p. 230-243.

DURHAM, Deborah. 2004. "Disappearing youth: youth as a social shifter in Botswana". American Ethnologist, v. 31, n. 4, p. 589-605.

HANCOCK, Anne Marie. 2007. "When multiplication doesn't equal quick addition: Examining intersectionality as a research paradigm”. Perspectives on Politics, n. 5, p. 63-79.

KROPFF, Laura. 2011. "Los jóvenes mapuche en Argentina: entre el circuito punk y las recuperaciones de tierras”. Alteridades, v. 21, n. 42, p. 77-89.

KROPFF, Laura. 2016. "Entre genealogías familiares y genealogías políticas: jóvenes en un proceso de comunalización mapuche en Argentina”. Mana - Estudos de Antropologia Social, v. 22, n. 2, p. 341-368.

KROPFF, Laura; PÉREZ, Pilar; CAÑUQUEO, Lorena; WALLACE, Julieta (Eds.). 2019. La tierra de los otros: la dimensión territorial del genocidio indígena en Río Negro y sus efectos en el presente. Viedma: Editorial UNRN.

LOWENHAUPT TSING, Anna. 2005. Friction: an ethnography of global connection. Princeton; Oxford: Princeton University Press.

MALKKI, Liisa H. 1992. "National Geographic: the rooting of peoples and the territorialization of national identity among scholars and refugees". Cultural Anthropology, n. 7, p. 24-44.

MARTINELLI, María Laura. 2019. "Procesos de territorialización y reservas indígenas en Norquincó: apuntes para la reconstrucción de las trayectorias de las comunidades de Ancalao y Cañumil, 1900-1930”. En: KROPFF, Laura; PÉREZ, Pilar; CAÑUQUEO, Lorena; WALLACE, Julieta (Eds.). La tierra de los otros: la dimensión territorial del genocidio indígena en Río Negro y sus efectos en el presente. Viedma: Editorial UNRN. p. 67-93.

MAUSS, Marcel. 1979 [1923-4]. "Ensayo sobre los dones. Motivo y forma del cambio en las sociedades primitivas”. En: MAUSS, Marcel. Sociología y Antropología. Madrid: Tecnos. p. 155-222.

ORGANIZACIÓN MAPUCHE “NEWENTUAYIÑ”; Pu WECHE FISKE MENUKO (JÓVENES MAPUCHE DE FISKE MENUCO). 2001. Postura Mapuche frente a la incorporación de "la variable indígena" en el Censo Nacional de Población y Vivienda 2001. Disponible en: http: / /www.mapuche.info/ mapuint/Newentuayin011000.html. Acceso en: 3 mayo 2016.

PACHECO DE OLIVEIRA, João. 1998. “Uma etnologia dos 'índios misturados'? Situação colonial, territorialização e fluxos culturais”. Mana, Estudos de Antropologia Social, v. 4, n. 1, p. 47-77. PÉREZ, Pilar. 2016. Archivos del silencio: estado, indígenas y violencia en Patagonia Central (18781941). Buenos Aires: Prometeo.

RADCLIFFE-BROWN, Alfred Reginald. 1929. “13. Age organization-terminology”. Man, n. 29, p. 21.

RUFFINI, Marta. 2001. "La cuestión de la tierra pública en Río Negro. Avances y perspectivas (Siglo XIX hasta la primera mitad del siglo XX)". Anuario del Centro de Estudios Históricos "Prof. Carlos 
S.A. Segreti”, n. 1.

SALAZAR, Noel; SMART, Alan. 2011. "Introduction, Anthropological Takes on (Im)Mobility". Identities: Global Studies in Culture and Power, n. 18, p. 1-9.

SHELLER, Mimi; URRY, John. 2006."The new mobilities paradigm”. Environment and Planning, n. 38, p. 207-226.

SVAMPA, Maristella. 1994. El dilema argentino: civilización o barbarie. De Sarmiento al revisionismo peronista. Buenos Aires: El Cielo por Asalto.

VIVALDI, Ana. 2011. "Stuck on a Muddy Road: Frictions of Mobility amongst Urban Toba in Northern Argentina". Identities: Global Studies in Culture and Power, n. 18, p. 599-619.

WILLIAMS, Raymond. 2001 [1973]. El campo y la ciudad. Buenos Aires: Paidós.

ZIZEK, Slavoj. 1998 [1993]. "Multiculturalismo o la lógica cultural del capitalismo multinacional”. En: JAMESON, Fredric; ZIZEK, Slavoj (Eds.). Estudios culturales: reflexiones sobre el multiculturalismo. Buenos Aires: Paidós. p. 137-187. 
Resumen: Pensar la movilidad de jóvenes indígenas como problema de investigación coloca en discusión tres dimensiones teóricas: la relación entre espacio y práctica social; la construcción de aboriginalidad en distintos contextos; y la edad como categoría que estructura la vida social. A partir de situaciones que involucran a jóvenes mapuche en la provincia de Río Negro (Argentina), este artículo se propone abordar la discusión poniendo en tensión la metafísica sedentaria inherente a los estados nacionales desde un enfoque centrado en la movilidad como práctica social que involucra fricciones múltiples. Así, se introducen, en primer lugar, las fijaciones y movilidades diferenciales producidas en el marco del proceso histórico de incorporación de la Patagonia al Estado argentino. Se trata de un proceso que supuso una configuración territorial específica en términos geopolíticos y productivos que, a la vez que consolidó la articulación entre el Estado, la nación y el territorio, instaló la dualidad entre lo rural y lo urbano. En segundo lugar, el artículo aborda las movilidades y las fricciones que se producen a nivel de prácticas cotidianas que se estructuran en la intersección entre edad, aboriginalidad y género, habilitando y disputando recorridos con efectos en la configuración territorial a nivel comunitario.

Palabras-clave: movilidad, aboriginalidad, edad, Estado, territorio.
Abstract: The research inquiry on young indigenous mobility offers up for discussion three theoretical dimensions: the relation between space and social practice; the construction of aboriginality in different contexts; and age as a category that structures social life. Taking into consideration different situations that involve young mapuche from the province of Río Negro (Argentina), this essay approaches the discussion by putting in tension the sedentarist metaphysic that is inherent to national states, with a perspective centered on mobility as social practice involving multiple frictions. The analysis deals, in first place, with the differential fixations and mobilities produced throughout the historical process of incorporation of Patagonia to the Argentinean state. This process involved a specific territorial configuration in geopolitical and productive terms: all at once, it strengthened the articulation between state, nation, and territory and settled the duality between the rural and the urban. In second place, the essay approaches mobilities and frictions that, produced in every day social practices, are structured at the intersection between age, aboriginality, and gender. These mobilities enable -and struggle towards-certain transits that have effects in community territorial configurations.

Keywords: mobility, aboriginality, age, state, territory. 